\title{
The Efficacy and Safety of Continuous Popliteal Sciatic Nerve Block for the Relief of Pain Associated with Critical Limb Ischemia: A Retrospective Study*
}

\author{
Atsushi Hashimoto ${ }^{\#}$, Hiroshi Ito, Yuko Sato, Yoshihiro Fujiwara \\ Department of Anesthesiology, Aichi Medical University, Nagakute, Japan. \\ Email: \#atsushih@aichi-med-u.ac.jp \\ Received October $11^{\text {th }}, 2013$; revised November $12^{\text {th }}, 2013$; accepted November $24^{\text {th }}, 2013$ \\ Copyright (C) 2013 Atsushi Hashimoto et al. This is an open access article distributed under the Creative Commons Attribution Li- \\ cense, which permits unrestricted use, distribution, and reproduction in any medium, provided the original work is properly cited.
}

\begin{abstract}
Background: Patients with critical limb ischemia (CLI) often suffer from severe pain. A continuous peripheral nerve block has been shown to provide effective analgesia for patients having lower limb surgery. We have been administering continuous sciatic nerve block (CSNB) for patients with CLI whose pain could not be relieved by other analgesic tools. The aim of this retrospective study was to investigate the efficacy and safety of CSNB for patients with CLI. Method: We retrospectively investigated 99 patients who received CSNB for the relief of severe pain in the lower limb associated with CLI. Patient demographics, neurological history, complications, and subjective evaluation of the effectiveness of CSNB were investigated from their clinical records. The distal tips of 108 catheters were cultured. Result: One hundred and seventy-two catheters were placed in 99 patients. More than $90 \%$ of the patients enjoyed considerable relief of severe pain. The analgesic effect of CSNB was greater in patients with older age and hemodialysis. Thirty-one catheters had positive bacterial colonization. However, no severe infectious complication was found. There was no relationship between the co-existence of diabetes and positive bacterial colonization. We encountered a patient with ASO and diabetes who suffered from persistent motor weakness and hypesthesia even after 3 months of CSNB placement. Conclusions: CSNB provided good pain control for patients with severe pain caused by CLI. Although catheters were frequently found to be colonized, infection at the catheter site was self-limiting even in patients with diabetes.
\end{abstract}

Keywords: Critical Limb Ischemia; Continuous Sciatic Nerve Block; Pain Control

\section{Introduction}

Critical limb ischemia (CLI) is defined as limb pain that occurs at rest, or impending limb loss that is caused by severe compromise of blood flow to affected extremity [1]. A recent Transatlantic Inter-Society Consensus [2] document on the management of peripheral arterial disease stresses the importance of optimizing pain control for all patients with CLI. Severe pain in patients with CLI may occur not only because of ischemia but also because of skin ulcer or gangrene. If the local infection ensues, pain can be exacerbated even further. Although several clinical options, including nonsteroidal anti-inflammatory medications (NSAIDs), narcotic medications, epidural analgesia, and spinal stimulations, are reported to relieve pain due to CLI [1], the application of these

${ }^{*}$ Conflict of interest: None declared

\#Corresponding author. options may be limited because of side effects or possible complications.

A continuous peripheral nerve block (CPNB) of the lower limb has been shown to provide multiple benefits for patients having lower limb surgery [3,4]. CPNB could be beneficial in that it is not associated with nuisance complications such as peptic ulcer, nausea and vomiting, hypotension, spinal hematoma. Although there are many studies involving various aspects of the postoperative pain control, there is no previous study about administration of CPNB to control severe pain due to CLI. In this retrospective study, we investigated the efficacy and safety of continuous sciatic nerve blocks (CSNB) in the treatment of severe pain associated with CLI.

\section{Methods}

After obtaining approval from Aichi Medical University 
Ethics Committee, we retrospectively investigated consecutive 99 patients who received CSNB for the relief of severe lower extremity pain associated with CLI at Aichi Medical University Hospital from January 2007 to December 2011. The severity of CLI was evaluated according to Fontaine classification before the administration of CSNB [5]. The degree of pain relief was evaluated by using subjective criteria (1: no relief of pain, 2: mild to moderate relief of pain, 3: considerable relief of pain, 4 : complete relief of pain) a few days after the initiation of CSNB. Duration of treatment, duration of catheter use, dose of local anesthetics, and complications were also recorded.

\subsection{Continuous Sciatic Nerve Blocks (CSNB)}

All sciatic catheters were placed under ultrasound guidance with aseptic technique according to standard precautions. Tuohy type, 18-gauge $10 \mathrm{~cm}$, needle was advanced under real-time ultrasound visualization. After confirming the tip of the needle is adjacent to the nerve, the catheter was threaded through the needle and sutured to the skin. The catheter was secured with a transparent adhesive dressing. The insertion site of the catheter was checked daily to see if there was any local sign of infection. On the termination of CSNB, some of the catheters were carefully and aseptically removed, and distal tip of the catheter was cut and cultured.

The continuous infusion of ropivacaine was initiated with a disposable elastomeric infusion pump (BaxterTM, Baxter international Inc., Deerfield, IL, USA). Dose of local anesthetic was titrated so that patients felt the pain relief was satisfactory while being able to move their foot voluntarily. If a patient was not satisfied with pain relief by CSNB, the replacement of sciatic catheter was considered. The administration of CSNB was terminated when the remission of pain or gangrene was attained.

\subsection{Statistical Analysis}

Chi-square test was used to compare the incidence of catheter colonization between the patients with and without diabetes, to determine the effect of patient characteristics (age, hemodialysis, and diabetes) on the effectiveness of CSNB. P $<0.05$ was considered to be statistically significant.

\section{Results}

From January 2007 to December 2011, 99 patients received CSNB for relief of severe lower limb pain due to CLI. All patients were classified as stage IV of Fontaine classification, which means all of them suffered from more or less skin ulcer and gangrene as shown in Figure 1. Patients' demographic data and their comorbidities are shown in Table 1.

One hundred and seventy-six catheters were used in 99 patients. The median duration of CSNB treatment was 28 days (range: 1 - 365 days). The median duration of the placement of each catheter was 18.0 days (range: 1 - 198 days) (Figure 2).

The degree of pain relief is shown in Figure 3. More than $90 \%$ of patients reported considerable pain relief. Pain relief was significantly more satisfactory in hemodialysis patients than those without it. Patients older than 70 years benefited from more pain relief than those younger than 70. Co-existence of diabetes did not affect the effectiveness of CSNB (Figure 4).

In all patients ropivacaine was used as a choice of local anesthetic. Most frequently used infusion rate and

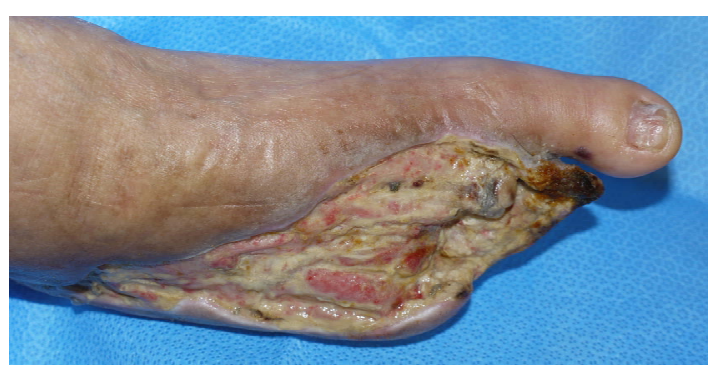

Figure 1. Necrotic foot in critical limb ischemia patient.

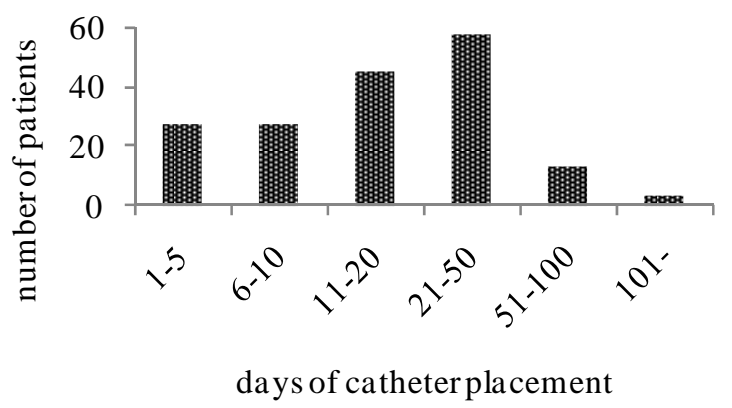

Figure 2. The duration of continuous sciatic nerve block catheter placement.

Table 1. Patients demographic data (mean \pm SD) and comorbidities (number of patients).

\begin{tabular}{cc}
\hline Sex (Male/Female) & $64 / 35$ \\
Age (years old) & $69.3 \pm 11.6$ \\
Height (cm) & $160.1 \pm 9.9$ \\
Weight $(\mathrm{kg})$ & $55.0 \pm 13.4$ \\
Diabetes mellitus & $52(54.7 \%)$ \\
Chronic renal failure with hemodialysis & $41(43.0 \%)$ \\
Arteriosclerosis obliterans & $64(67.4 \%)$ \\
\hline
\end{tabular}




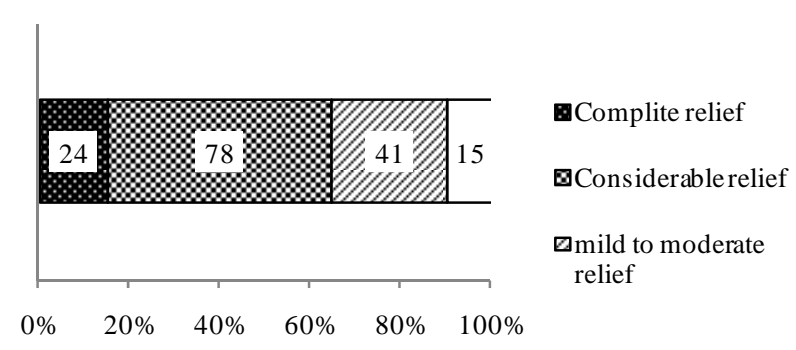

Figure 3. The degree of pain relief. Pain associated with critical limb ischemia was relieved more than $90 \%$ patients.
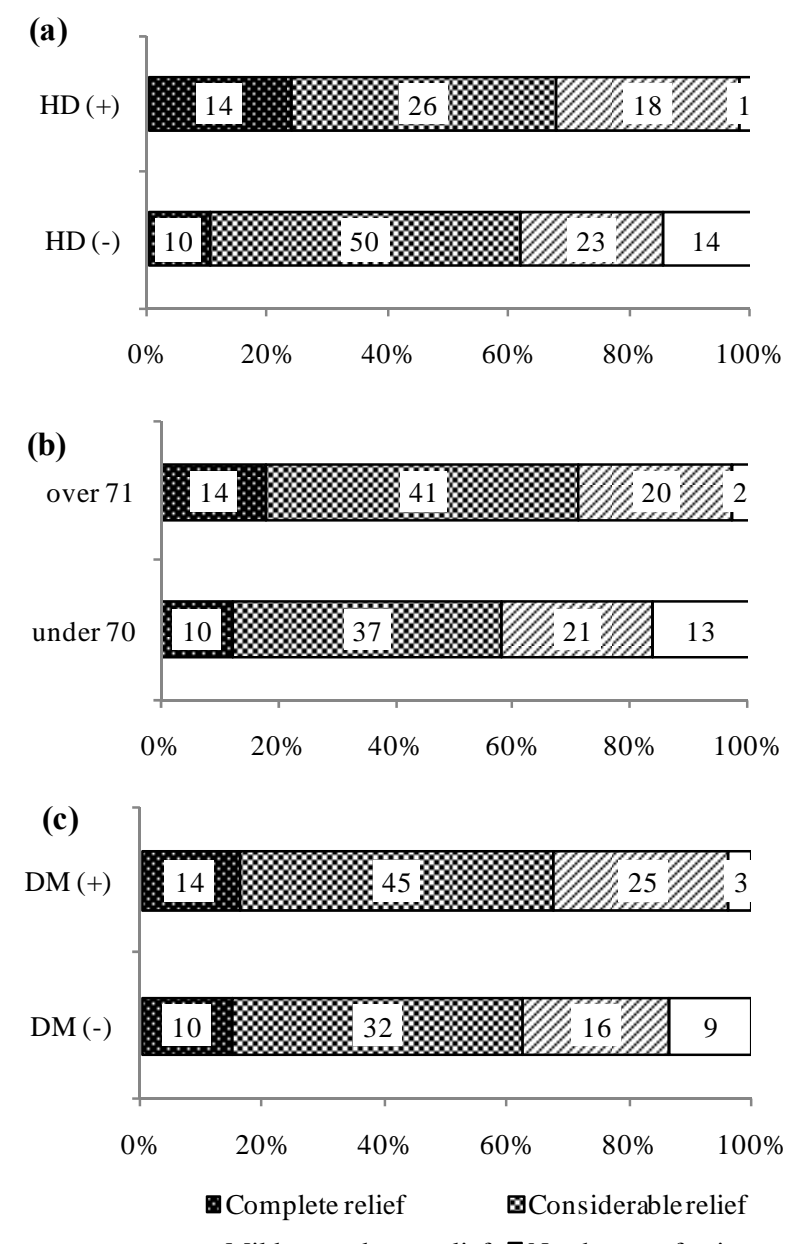

Figure 4. The effect of patient characteristics ((a) hemodialysys (HD), (b) age and (c) diabetes (DM)) on the effective of continuous sciatic nerve block (CSNB). Pain relief was significantly more satisfactory in $\mathrm{HD}$ patients $(P=0.009$; HD $(+)$ vs. HD $(-))$. Patients older than 70 years benefited from more pain relief $(P=0.031$; years over 71 vs. under 70). Co-existence of $D M$ did not affect the effectiveness of CSNB (P = 0.149; DM (+) vs. DM (-)).

concentration of ropivacaine was $5 \mathrm{ml} / \mathrm{h}$ and $0.15 \%$ (Table 2). The distal tips of 108 catheters were cultured. Thirty-three catheters (31\% of cultured catheters) had positive bacterial colonization. The bacterial species most frequently found were staphylococcus (Table 3). Eleven catheters were removed during the course of therapy because of local infectious sign such as redness, tenderness, or swelling. However, severe local infection requiring surgical drainage was not encountered. The presence of diabetes did not affect the incidence of colonization (Table 4). Fifty-four patients received lower extremity amputation during the CSNB treatment. After the amputation, CSNB was continued until the stump pain was dismissed. Sixty-five patients received artery bypass operation during the CSNB treatment. Nine patients died of their comorbidities during CSNB treatment.

We experienced one case of severe persistent nerve injury in a patient aged 73 years old with ASO and diabetes. During the insertion of the catheter, he complained of neither paresthesia nor pain. Ropivacaine $0.5 \%$ was

Table 2. The distribution of ropivacaine concentration and the rate of administration for continuous sciatic nerve block.

\begin{tabular}{ccc}
\hline Concentration of ropivacaine & Rate $(\mathrm{ml} / \mathrm{hr})$ & cases \\
\hline $0.05 \%$ & 5 & 1 \\
$0.10 \%$ & 3 & 3 \\
$0.10 \%$ & 5 & 11 \\
$0.125 \%$ & 5 & 3 \\
$0.15 \%$ & 2 & 1 \\
$0.15 \%$ & 3 & 4 \\
$0.15 \%$ & 5 & 30 \\
$0.2 \%$ & 3 & 5 \\
$0.2 \%$ & 5 & 25 \\
\hline
\end{tabular}

Table 3. Bacterial species found in catheters culture.

\begin{tabular}{cc}
\hline Bacteria species & Number of catheters \\
\hline Staphylococcus & 22 \\
Enterococcus & 4 \\
Psudmonas aerogenes & 2 \\
Cortnebacterium & 2 \\
Others & 3 \\
\hline
\end{tabular}

Table 4. Incidence of Cather colonization with or without diabetes.

\begin{tabular}{ccc}
\hline & Diabetes $(+)$ & Diabetes $(-)$ \\
\hline Colonization $(+)$ & 18 & 42 \\
Colonization $(-)$ & 42 & 32 \\
\hline
\end{tabular}


initially administered to block sciatic nerve and CSNB was initiated. Since complete motor and sensory deficit in the distribution of sciatic nerve did not resolve for 2 days, CSNB was terminated and the catheter was removed. However complete motor and sensory deficit persisted for more than 3 month until he died of sepsis. Nerve conduction test was made after the nerve injury. Nerve conduction velocity (NCV) on the damaged leg was not detected, while NCV on the contralateral leg was reduced.

\section{Discussion}

This retrospective study showed that the CSNB is an effective tool to relieve severe pain associated with CLI. All patients involved in this study suffered from skinulcer or gangrene. Thus, the pain experienced by these patients was clearly due to tissue damage or inflammation rather than simply due to ischemia. The mechanisms of ischemic pain in CLI are still not fully understood. Inadequate oxygen supply to the tissues can produce pain by causing the release of chemical substances, such as histamine, potassium ions, hydrogen ions, and bradykinin. When CLI is accompanied by the skin ulcer or gangrene, the release of these chemical substances can be even greater. Chemical substances released from ischemic tissue sensitize the nociceptor of sensory afferent fibers, which result in primary hyperalgesia. We speculate that continuous sciatic nerve block produced considerable pain relief in patients with CLI by attenuating the conduction of noxious stimuli via primary afferent nerve fibers. Interestingly, Ruger et al. [6] suggested that neuropathic component contributes to ischemic pain in patients with CLI. In fact, pain relief was not adequate in a few patients even after CSNB treatment. Supplementation with other methods such as opioids or pregabalin may be needed for these patients.

We found that CSNB provides better pain relief in older patients and hemodialysis patients. Hanks et al. [7] recently reported that the duration of single-shot sciatic nerve block was longer in older patients. Aging can impact on the response of peripheral nerve to local anesthetics by modulating the functional and electrophysiological properties of it. About 16 percent of hemodialysis patients suffer from uremic polyneuropathy [8], which may also modulate the susceptibility of peripheral nerve to local anesthetics. Although the co-existence of diabetes has been reported to result in higher success rate for supraclavicular brachial plexus block [9], it did not affect the analgesic effect of CSNB in our study. The difference of the type of nerve block may explain the discrepancy of the results of these studies.

The goals of treating CLI are to relieve ischemic pain, heal ischemic ulcer, prevent limb loss, improve patient function and QOL, and prolong survival. Therefore pain control is the most important therapy until revascularization or amputation of the limb. Epidural analgesia is an effective tool to control severe pain. However, we were very reluctant to administer epidural analgesia for these patients because they are frequently under anticoagulation or antiplatelet therapy. Although opioid is another way to control severe pain, it is often associated with side effects such as nausea and vomiting, sedation, hallucination, or respiratory depression. The prevalence of renal insufficiency or cognitive dysfunction in patients with CLI sometimes makes it difficult to administer opioids to patients with CLI. Our case series are the first report describing the application of CSNB for pain relief in patients with CLI. Ninety percent of our patients enjoyed considerable pain relief during CSNB treatment. In many patients, considerable pain relief is significantly improved QOL by resolving insomnolence. We suggest that CSNB is an effective tool to relieve severe pain in patients with CLI.

A recent review of infectious risk related to postoperative CPNB highlighted a frequency of colonization ranging between $23 \%$ and $57 \%$ and reported incidence of local infection between $0 \%$ and 3.2\% [10]. CSNB for CLI has to be continued much longer than postoperative analgesia. In this study, the median duration of CSNB treatment was 29 days and median duration of each catheter was 18 days. CPNB of duration longer than $48 \mathrm{~h}$ has been reported to be an independent risk factor for local inflammation and catheter-related local infection [3] or catheter colonization [11]. Fortunately, no severe infectious complication was found in the present study. Although thirty-three catheters (31\% of cultured catheters) had positive bacterial colonization, Staphylococcus, which was the most frequently found bacterial species, may be a contamination of the catheter on removal despite aseptic condition. Capdevilla et al. reported the site of catheter insertion could be another potential risk factor for bacterial colonization [10]. The femoral or axillary sites are associated with higher rate of catheter bacterial colonization, whereas the popliteal catheter has a reduced rate. Our results confirm that the popliteal catheter is not associated with increased risk of infection despite the long duration of catheter placement.

Aveline et al. reported diabetic patients were identified in their study as higher-risk patients for perineural catheter colonization [11]. They found $10 \%$ of catheters were colonized. Compared with their study (diabetes/total patients, 60/747), more diabetic patients were involved in our study. It might be the reason why the incidence of colonization in our study was higher than in their study.

Unfortunately, we had a case of severe nerve injury associated with CSNB in the patient with diabetes. We 
confirmed the injection of local anesthetics was not intraneural under real time ultrasound guidance. After the nerve damage was manifested, nerve conduction velocity (NCV) on bilateral legs was examined. As the NCV of contralateral leg was also found to be moderately reduced, the preexistence of significant nerve dysfunction was suspected. CLI patients' underlying diabetic neuropathy and ischemic damage of the nerve may have made their nerves more susceptible to local anesthetic toxicity [12]. As many of patients with CLI potentially have intrinsic neuropathy, the safety of administering CPNB for patients with CLI should be further investigated.

In conclusion, the result of our retrospective study showed that CSNB provided good pain control in patients with CLI. Although about $30 \%$ of catheters were found to be colonized, infection at the catheter site was easily controlled or self-limiting. We experienced permanent nerve damage in a patient with preexisting neuropathy. The effect of this technique on neurological complications in patients with preexisting neuropathy should be further examined.

\section{Acknowledgements}

This research received no specific grant from any funding agency in the public, commercial, or not-for-profit sectors.

\section{REFERENCES}

[1] V. N. Varu, M. E. Hogg and M. R. Kibbe, “Critical Limb Ischemia,” Journal of Vascular Surgery, Vol. 51, No. 1, 2010, pp. 230-241. http://dx.doi.org/10.1016/j.jvs.2009.08.073

[2] L. Norgren, W. R. Hiatt, J. A. Dormandy, M. R. Nehler, K. A. Harris, F. G. Fowkes and TASC II Working Group, "Inter-Society Consensus for the Management of Peripheral Arterial Disease (TASC II)," Journal of Vascular Surgery, Vol. 45, No. S1, 2007, pp. S5-S67. http://dx.doi.org/10.1016\%2Fj.jvs.2006.12.037

[3] X. Capdevila, P. Pirat, S. Bringuier, E. Gaertner, F. Singelyn, N. Bernard, O. Choquet, H. Bouaziz, F. Bonnet and French Study Group on Continuous Peripheral Nerve Blocks, "Continuous Peripheral Nerve Blocks in Hospital Wards after Orthopedic Surgery: A Multicenter Prospective Analysis of the Quality of Postoperative Analgesia and Complications in 1416 Patients,” Anesthesiology, Vol. 103, No. 5, 2005, pp. 1035-1045. http://dx.doi.org/10.1097/00000542-200511000-00018

[4] B. M. Ilfeld and F. K. Enneking, "Continuous Peripheral Nerve Blocks at Home: A Review,” Anesthesia \& Analgesia, Vol. 100, No. 6, 2005, pp. 1822-1833. http://dx.doi.org/10.1213/01.ANE.0000151719.26785.86

[5] R. Fontaine, M. Kim and R. Kieny, "Surgical Treatment of Peripheral Circulation Disorders,” Helvetica Chimica Acta, Vol. 21, No. 5-6, 1954, pp. 499-533.

[6] L. J. Ruger, D. Irnich, T. N. Abahji, A. Crispin, U. Hoffmann and P. M. Lang, "Characteristics of Chronic Ischemic Pain in Patients with Peripheral Arterial Disease," Pain, Vol. 139, No. 1, 2008, pp. 201-208. http://dx.doi.org/10.1016/j.pain.2008.03.027

[7] R. K. Hanks, R. Pietrobon, K. C. Nielsen, S. M. Steele, M. Tucker, D. S. Warner, K. P. King and S. M. Klein, "The Effect of Age on Sciatic Nerve Block Duration,” Anesthesia \& Analgesia, Vol. 102, No. 2, 2006, pp. 588-592. http://dx.doi.org/10.1213/01.ane.0000189552.85175.db

[8] E. Mambelli, M. Barrella, M. G. Facchini, E. Mancini, C. Sicuso, S. Bainotti, M. Formica and A. Santoro, "The Prevalence of Peripheral Neuropathy in Hemodialysis Patients,” Anesthesia \& Analgesia, Vol. 77, No. 6, 2012, pp. 468-475. http://dx.doi.org/10.5414/CN107188

[9] R. E. Gebhard, K. C. Nielsen, R. Pietrobon, A. Missair and B. A. Williams, "Diabetes Mellitus, Independent of Body Mass Index, Is Associated with a 'Higher Success' Rate for Supraclavicular Brachial Plexus Blocks," Regional Anesthesia \& Pain Medicine, Vol. 34, No. 5, 2009, pp. 404-407. http://dx.doi.org/10.1097/AAP.0b013e3181ada58d

[10] X. Capdevila, S. Bringuier and A. Borgeat, "Infectious Risk of Continuous Peripheral Nerve Blocks,” Anesthesiology, Vol. 110, No. 1, 2009, pp. 182-188. http://dx.doi.org/10.1097/ALN.0b013e318190bd5b

[11] C. Aveline, H. Le Hetet, A. Le Roux, P. Vautier, J. F. Gautier, F. Cognet, P. Auger and F. Bonnet, "Perineural Ultrasound-Guided Catheter Bacterial Colonization: A Prospective Evaluation in 747 Cases,” Regional Anesthesia \& Pain Medicine, Vol. 36, No. 6, 2011, pp. 579-584. http://dx.doi.org/10.1097/AAP.0b013e31822e665a

[12] B. A. Williams, B. B. Murinson, B. R. Grable and S. L. Orebaugh, "Future Considerations for Pharmacologic Adjuvants in Single-Injection Peripheral Nerve Blocks for Patients with Diabetes Mellitus,” Regional Anesthesia \& Pain Medicine, Vol. 34, No. 5, 2009, pp. 445-457. http://dx.doi.org/10.1097/AAP.0b013e3181ac9e42 